\title{
Simultaneous Ipsilateral Vascularized Lymph Node Transplantation and Contralateral Lymphovenous Anastomosis in Bilateral Extremity Lymphedema with Different Severities
}

\author{
M.-H. Cheng, MD, MBA ${ }^{1,2}$, R. Tee, MBBS, PhD ${ }^{1,2}$, C. Chen, $\mathrm{MD}^{3}$, C.-Y. Lin, MSc ${ }^{1}$, and \\ M. Pappalardo, $\mathrm{MD}^{4}$ \\ ${ }^{1}$ Department of Plastic and Reconstructive Surgery, Chang Gung Memorial Hospital, College of Medicine, Chang Gung \\ University, Taoyuan, Taiwan; ${ }^{2}$ Center for Tissue Engineering, Chang Gung Memorial Hospital, Taoyuan, Taiwan; \\ ${ }^{3}$ Department of General Surgery, Cedars Sinai Medical Center, Los Angeles, CA; ${ }^{4}$ Plastic and Reconstructive Surgery, \\ Department of Surgical, Oncological and Oral Sciences, University of Palermo, Palermo, Italy
}

\begin{abstract}
Background. Extremity lymphedema can occur bilaterally with different severities on each side. The aim of this study is to investigate the treatment outcomes of such patients with bilateral extremity lymphedema of different severities.

Patients and Methods. Between 2013 and 2017, patients with bilateral extremity lymphedema of different severities according to the Taiwan Lymphoscintigraphy Staging (TLS) system were retrospectively reviewed. Ipsilateral vascularized lymph node transplantation (VLNT) was indicated in TLS total obstruction and contralateral lymphovenous anastomosis (LVA) in TLS partial obstruction with patent lymphatic vessels on indocyanine green lymphography. Outcomes were assessed using circumference improvement, frequency of cellulitis, and lymphedemaspecific quality of life (LYMQoL) questionnaires.

Results. A total of 10 patients with bilateral extremity lymphedema with median age of 63 (range 12-75) years were included. The median symptom duration of the lymphedematous limb was 60 (range 36-168) months and 12 (range 1-60) months in the VLNT and LVA group, respectively $(p<0.05)$. At average follow-up of 37.5 (range 14-58) months, the average limb circumference
\end{abstract}

(C) The Author(s) 2020

First Received: 23 October 2019;

Published Online: 18 June 2020

M.-H. Cheng, MD, MBA

e-mail: minghueicheng@gmail.com;

minghuei@adm.cgmh.org.tw improvement was 2.4 (range -3.3 to 7.8 ) $\mathrm{cm}$ in the VLNT group and 2.3 (range $0.3-7$ ) $\mathrm{cm}$ in the LVA group $(p=1$ ). The median episodes of cellulitis decreased significantly from 4 to 0.5 and 1 to 0 times/year in the VLNT and LVA group, respectively $(p=0.02, p=0.06)$. The overall LYMQoL score improved from 4.5 preoperatively to 7.5 postoperatively $(p<0.01)$.

Conclusions. Limb-specific VLNT and LVA selected by TLS effectively treated bilateral extremity lymphedema with different severities.

Secondary extremity lymphedema is a burdensome sequela experienced by cancer survivors. ${ }^{1}$ Lymphedema management typically consists of complex decongestive therapy, which is only partially effective and does not prevent the progression of extremity lymphedema. ${ }^{2}$ Advances in lymphedema microsurgery including lymphovenous anastomosis (LVA) ${ }^{3}$ and vascularized lymph node transplantation (VLNT) ${ }^{4}$ have yielded promising outcomes over the last decade. Both techniques redirect stagnant lymph in the lymphedematous limb to the venous system, which is achieved by directly shunting the lymph into a subdermal venule in the LVA technique or by bypassing lymph through transplanted lymph nodes in VLNT. ${ }^{5,6}$

Independently, the selection of LVA versus VLNT for treatment of extremity lymphedema has been the subject of academic debate across centers owing to a variance in disease assessments and plans, including the presentations of severity, staging/grading systems, insurance limitations, patient preferences, and even surgeon experience. ${ }^{6}$ The 
general consensus of experts on lymphedema microsurgery is that LVA is more effective in the earlier stages of lymphedema while VLNT is commonly indicated in more advanced cases. ${ }^{7}$ A more complex scenario is when extremity lymphedema develops simultaneously in both limbs but with different stages of severity in each, representing a unique challenge for procedure selection. The first treatment of primary bilateral extremity lymphedema by LVA in one limb and VLNT in the other limb for different severities of lymphedema was reported in $2014 .^{8}$ An objective tool to evaluate the severity of extremity lymphedema and select an appropriate surgical treatment is Cheng's Lymphedema Grading (CLG), which utilizes clinical circumferential measurement to assess the normal extremity as a control in unilateral extremity lymphedema., ${ }^{9,10}$ Consequently, as understanding of the pathophysiology of extremity lymphedema deepens, the evaluations and treatments of different lymphedemas have been extended and the applicability of current assessment tools also needs to be explored. However, there is a lack of reports and guidelines for evaluating and selecting the appropriate surgical treatment for patients with bilateral extremity lymphedema. This condition is inherently an important form of clinical lymphedema that can benefit from investigative guidelines, as it is inappropriate to assume that patients with differing limb lymphedema severities can be adequately treated with a single surgical procedure.

The aim of this study is to investigate the patient selection and treatment outcomes of ipsilateral VLNT and contralateral LVA for individual lymphedematous limb in patients with bilateral extremity lymphedema of different severities.

\section{PATIENTS AND METHODS}

This single-institutional retrospective study of patients with bilateral extremity lymphedema who underwent ipsilateral VLNT and contralateral LVA between November 2013 and October 2018 was approved by the Chang Gung Memorial Hospital Institutional Review Board (IRB: 201800600B0). Lymphoscintigraphy and indocyanine green (ICG) lymphography were parts of the standard preoperative work-up and assisted in evaluating extremity lymphedema severity and in guiding the selection of the appropriate surgical procedures. Magnetic resonance imaging (MRI) was used to evaluate the donor-site lymph node basins. Doppler ultrasound was used to assess the recipient-site vessels.

Patients with bilateral extremity lymphedema of different severities in both limbs according to the Taiwan Lymphoscintigraphy Staging (TLS) system were indicated to undergo ipsilateral VLNT (VLNT group) and contralateral LVA (LVA group). ${ }^{8,9,11}$ Lymphedematous limbs with longer symptom duration ( $>5$ years) and that were stage P-3 or total obstruction stages T-4, T-5, or T-6 according to the Taiwan Lymphoscintigraphy Staging system but did not have patent lymphatic ducts on ICG lymphography underwent vascularized submental lymph node (VSLN) flap transfer. ${ }^{8,9,11,12}$ Lymphedematous limbs with relatively short-term symptoms $(<5$ years $)$, partial obstruction in Taiwan Lymphoscintigraphy stages P-1, P-2, or P-3, and patent lymphatic ducts identified on ICG lymphography were selected for LVA. ${ }^{13,14}$ Patients with unilateral extremity lymphedema and those with bilateral lymphedema who underwent the same procedures bilaterally for both limbs were excluded. ${ }^{8}$

The prospectively collected data included patient demographics, lymphedema etiology, duration of symptoms prior to treatment, compliance with complex decongestive therapy, TLS severity of both limbs, and episodes of cellulitis. Compliance with complex decongestive therapy was defined as use of grade 2 compression garments for at least $12 \mathrm{~h}$ per day in addition to daily manual lymphatic drainage. Patient limb circumferential measurements were taken preoperatively and postoperatively at every clinical visit and were measured at $10 \mathrm{~cm}$ above and below the elbow joint for the upper limbs and $15 \mathrm{~cm}$ proximal and distal to the patella for the lower limbs. While the circumferential measurements were utilized in surgical outcome comparisons, they were not incorporated into circumferential differences or circumferential reduction rate, since there was no "healthy" limb to serve as a baseline. ${ }^{10}$

Changes in the circumference of each extremity from before to after the operation were documented. Preoperative versus postoperative comparisons were also carried out for the number of episodes of cellulitis per year.

\section{Lymphedema-Specific Quality of Life (LYMQoL) Questionnaire}

Upper or lower extremity lymphedema-specific quality of life (LYMQoL) questionnaires were administered both preoperatively and at 12 months postoperatively to patients receiving upper or lower limb treatment, respectively. ${ }^{9-11,15,16}$ The questionnaires consisted of 27 questions (upper extremity) or 28 questions (lower extremity) covering four domains (function, appearance, symptoms, and mood). A lower score in each domain corresponds to better QoL in that aspect. The overall patient QoL was scored on a scale of 0 to 10 , with 10 being the best QoL score (inverse to the individualized domains of LYMQoL). 
Surgical Techniques

A VSLN flap was harvested by a modified technique with partial preservation of the medial platysma, as described previously. ${ }^{17,18}$ The dorsal wrist and ankle were the preferred recipient sites of the upper and lower extremity transfers, respectively, as described by Cheng et al. $^{4,17}$ Key maneuvers included careful microscopic dissection of the branches of the marginal mandibular nerve, sparing of the medial platysma, $5 \mathrm{~cm}$ in width, to avoid marginal mandibular nerve pseudoparalysis, ${ }^{18}$ vigilance for regional vascular anatomical variations, and optimization in harvesting the maximal number of submental lymph nodes available in the region. ${ }^{19}$

The side-to-end LVA technique performed by the senior author (M.-H.C.) was previously described with preoperative ICG lymphography evaluation of the patent lymphatic vessels available. ${ }^{13,14}$ Use of ultrasound for the evaluation of the lymphatic vessels and recipient venules was not available at our hospital. ${ }^{20,21}$ One or two side-to-end anastomoses between a lymphatic channel and subdermal venule were executed preferentially under 42× magnification using a Mitaka MM50 microscope (Kohki Co, Ltd., Tokyo, Japan) for early-grade lymphedematous limbs. Anastomoses were performed using 11-0 Nylon sutures (Ethicon, New Brunswick, NJ). ICG lymphography was used to map the lymphatic ducts preoperatively and intraoperatively to confirm anastomosis patency. ${ }^{13,22}$

\section{Postoperative Care}

After simultaneously receiving VLNT and LVA, patients were asked to comply with a standardized 2-week rehabilitation program upon discharge, consisting of progressive muscle strength training and reverse manual proximal-to-distal lymphatic drainage. Patients did not wear any compression garments or bandages postoperatively on either limb.

\section{Statistics}

The data are presented as median and range for continuous variables. The statistical analyses were performed using SPSS 21.0 statistical software (SPSS, Inc., Chicago, Ill.). The nonparametric Mann-Whitney $U$ test was used for comparisons between the VLNT and LVA groups. Preoperative and postoperative differences were analyzed by Wilcoxon matched-pairs signed-rank tests. A $p$ value $\leq 0.05$ was considered statistically significant.

\section{RESULTS}

A total of ten patients with bilateral extremity lymphedema with different severities between the lymphedematous limbs who underwent ipsilateral VLNT and contralateral LVA at the same time were included. All ten patients were female, with median age of 63 (range 12-75) years preoperatively (Table 1). Three patients had primary bilateral lower extremity lymphedema. Five patients with bilateral lower extremity lymphedema received adjuvant radiation therapy to the pelvis but presented with lower extremity lymphedema of different severities. Two bilateral breast cancer patients received adjuvant radiation therapy after axillary lymph node dissection in unilateral extremity, which was subjected to a VLNT procedure. In the LVA group, partial obstruction of TLS P-1 was present in four limbs, while P-2 was observed in six limbs. In the VLNT group, total obstruction of TLS $\mathrm{T}-4$ was present in four limbs, and T-5 was observed in six limbs. The average symptom duration and preoperative duration of CDT were 60 (range 36-168) months and 12 (range 0-24) months in the VLNT group, and 12 (range 1-60) months and 4.8 (range 0-24) months in the LVA group ( $p<0.05$ and $<0.05$, respectively) (Table 2 ).

\section{Response to Lymphedema Microsurgeries}

Ten limbs underwent a successful VSLN flap, to the ankle in eight limbs and the wrist in two limbs, with a $100 \%$ flap success rate. One of the VSLN flaps required reexploration due to venous congestion, which was successfully salvaged with a vein reanastomosis (case 3 ). The average preoperative body mass index (BMI) of 25.9 (range 19.9-30.6) $\mathrm{kg} / \mathrm{m}^{2}$ was statistically improved to $23.7 \mathrm{~kg} / \mathrm{m}^{2}$ (range $19.7-29.9$ ) $\mathrm{kg} / \mathrm{m}^{2}$ postoperatively $(p=0.01)$. All patients who received a VSLN flap did not develop facial lymphedema or marginal mandibular nerve palsy. One side-to-end anastomosis was performed in nine extremities and two anastomoses were performed in one extremity in the LVA group, giving a mean of 1.1 anastomoses. There were no complications associated with any of the LVAs.

One patient (case 7) developed a pelvic recurrence 10 months postoperatively. At an average follow-up time of 37.5 (range 14-58) months, the average limb circumference decreased by 2.4 (range -3.3 to 7.8 ) $\mathrm{cm}$ and 2.3 (range 0.3-7) $\mathrm{cm}$ in the VLNT and LVA group, respectively $(p=1)$ (Table 3$)$. Six of the eight $(75 \%)$ limbs in the VLNT group and eight of eight (100\%) in the LVA group showed circumferential improvement after operations, respectively (Table 3), excluding one case with recurrence and one case younger than 12 years old. Case 3 who had an unsatisfactory outcome in the limb underwent VLNT due 
TABLE 1 Demographics and etiology of ten patients with bilateral extremity lymphedema

\begin{tabular}{llll}
\hline & Age (years) & Preop. BMI $\left(\mathrm{kg} / \mathrm{m}^{2}\right)$ & Etiology (change to radiation, chemotherapy) \\
\hline Case 1 & 75 & 27.4 & Cervical cancer with hysterectomy and RT \\
Case 2 & 64 & 30.0 & Endometrial cancer with hysterectomy and PLND + RT + CT \\
Case 3 & 71 & 30.6 & Endometrial cancer with hysterectomy and BSO + LND + RT + CT \\
Case 4 & 60 & 19.9 & Right breast cancer with mastectomy and ALND + RT \\
& & & Left breast cancer with mastectomy and CT \\
Case 5 & 61 & & Right breast cancer with mastectomy and ALND + CT + RT \\
Case 6 & 63 & 25.4 & Left breast cancer with mastectomy and ALND + CT \\
Case 7 & 63 & 24.2 & Endometrial cancer with hysterectomy + BSO + PLND + RT + CT \\
Case 8 & 63 & 22.1 & Primary lymphedema \\
Case 9 & 60 & 26.3 & Cervical cancer with hysterectomy and RT \\
Case 10 & 12 & 26.8 & Primary lymphedema \\
Median (range) & $63(12-75)$ & $25.9(19.9-30.6)$ & Primary lymphedema \\
\hline
\end{tabular}

$R T$ radiotherapy, $A L N D$ axillary lymph node dissection, $C T$ chemotherapy, $B S O$ bilateral salpingo-oophorectomy, $P L N D$ pelvic lymph node dissection

TABLE 2 Characteristics of bilateral extremity lymphedema subjected to ipsilateral vascularized lymph node transplantations and contralateral lymphovenous anastomoses

\begin{tabular}{|c|c|c|c|c|c|c|c|c|}
\hline \multirow[t]{2}{*}{ Case no. (limb) } & \multirow[t]{2}{*}{ Limbs affected } & \multirow[t]{2}{*}{ Side } & \multirow[t]{2}{*}{ Procedure } & \multicolumn{2}{|l|}{ Duration } & \multicolumn{2}{|l|}{ Lymphoscintigraphy } & \multirow{2}{*}{$\begin{array}{l}\text { ICG } \\
\text { Patent lymphatic } \\
\text { ducts (yes/no) }\end{array}$} \\
\hline & & & & Symptoms (months) & CDT (months) & Pattern & Stage & \\
\hline 1 & Lower & $\mathrm{L}$ & LVA & 12 & 0 & Partial obstruction & P-1 & Yes \\
\hline 2 & Lower & $\mathrm{R}$ & LVA & 60 & 24 & Partial obstruction & $\mathrm{P}-2$ & Yes \\
\hline 3 & Lower & $\mathrm{R}$ & LVA & 36 & 0 & Partial obstruction & P-1 & Yes \\
\hline 4 & Upper & $\mathrm{L}$ & LVA & 1 & 0 & Partial obstruction & $\mathrm{P}-2$ & Yes \\
\hline 5 & Upper & $\mathrm{L}$ & LVA & 1 & 0 & Partial obstruction & $\mathrm{P}-2$ & Yes \\
\hline 6 & Lower & $\mathrm{R}$ & LVA & 14 & 12 & Partial obstruction & P-2 & Yes \\
\hline 7 & Lower & $\mathrm{L}$ & LVA & 6 & 0 & Partial obstruction & $\mathrm{P}-1$ & Yes \\
\hline 8 & Lower & $\mathrm{L}$ & LVA & 3 & 0 & Partial obstruction & P-1 & Yes \\
\hline 9 & Lower & $\mathrm{R}$ & LVA & 3 & 0 & Partial obstruction & $\mathrm{P}-2$ & Yes \\
\hline 10 & Lower & $\mathrm{R}$ & LVA & 120 & 36 & Partial obstruction & $\mathrm{P}-2$ & Yes \\
\hline \multicolumn{4}{|c|}{ Subtotal of LVA, median (range) } & $12 *(1-60)$ & $4.8 *(0-24)$ & & & \\
\hline 1 & Lower & $\mathrm{R}$ & VLNT & 168 & 6 & Total obstruction & $\mathrm{T}-5$ & No \\
\hline 2 & Lower & $\mathrm{L}$ & VLNT & 60 & 24 & Total obstruction & $\mathrm{T}-4$ & No \\
\hline 3 & Lower & $\mathrm{L}$ & VLNT & 36 & 0 & Total obstruction & $\mathrm{T}-5$ & No \\
\hline 4 & Upper & $\mathrm{R}$ & VLNT & 36 & 12 & Total obstruction & $\mathrm{T}-5$ & No \\
\hline 5 & Upper & $\mathrm{R}$ & VLNT & 84 & 24 & Total obstruction & $\mathrm{T}-5$ & No \\
\hline 6 & Lower & $\mathrm{L}$ & VLNT & 36 & 24 & Total obstruction & $\mathrm{T}-4$ & No \\
\hline 7 & Lower & $\mathrm{R}$ & VLNT & 36 & 12 & Total obstruction & $\mathrm{T}-4$ & No \\
\hline 8 & Lower & $\mathrm{R}$ & VLNT & 12 & 6 & Total obstruction & $\mathrm{T}-5$ & No \\
\hline 9 & Lower & $\mathrm{L}$ & VLNT & 240 & 240 & Total obstruction & $\mathrm{T}-5$ & No \\
\hline 10 & Lower & $\mathrm{L}$ & VLNT & 120 & 36 & Total obstruction & $\mathrm{T}-4$ & No \\
\hline \multicolumn{4}{|c|}{ Subtotal of VLNT, median (range) } & $60 *(36-168)$ & $12 *(0-24)$ & & & \\
\hline
\end{tabular}

LVA lymphovenous anastomosis, $C D T$ complex decongestive therapy, $V L N T$ vascularized lymph node transplantation

*Statistically significant, $p<0.05$ 


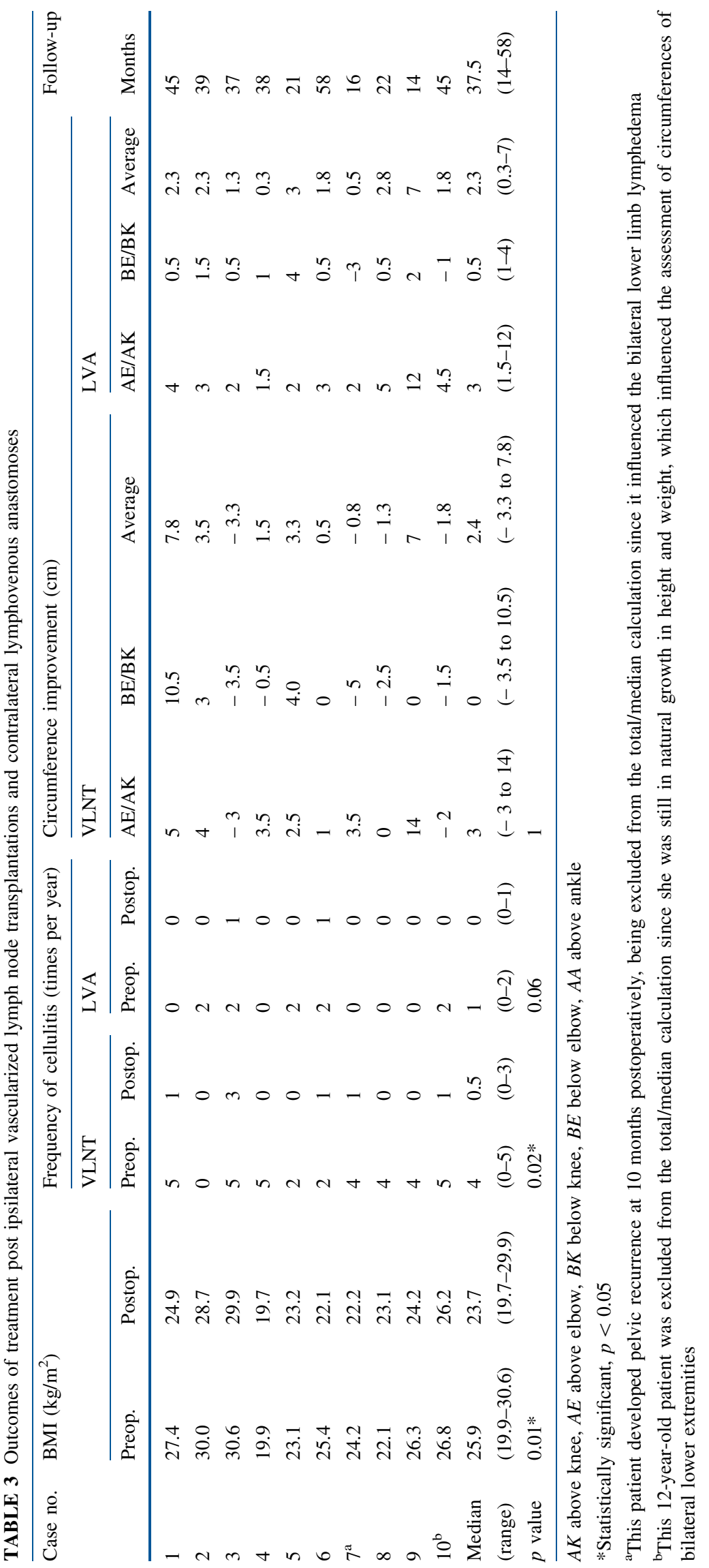


to venous compromise, but not in the LVA limb (Table 3). The average number of episodes of cellulitis decreased significantly from 4 (range $0-5$ ) to 0.5 (range 0-3) times/ year $(p=0.02)$ in the VLNT group, and from 1 (range $0-2$ ) to 0 (range $0-1)$ times/year $(p=0.06)$ in the LVA group.

\section{Improvement in $L Y M Q o L$}

The preoperative LYMQoL scores showed high levels of functional impairment and morbidity with median scores of 34 (range 30-38) in function, 28 (range 19-28) in appearance, 20 (range 18-24) in symptoms, and 24 (range 22-24) in mood. At mean follow-up of 12 months, the scores of all domains improved, with 18 (range 16-25) in function $(p<0.01), 10$ (range $8-20)$ in appearance $(p<0.01), 10$ (range $8-18)$ in symptoms $(p<0.01$ ), and 10 (range 7-18) in mood $(p<0.01)$. The overall LYMQoL score showed improvement from a score of 4.5 (range 2-5) preoperatively to 7.5 (range 6-8) postoperatively $(p<0.01)$ (Fig. 1) (Table 4).

\section{DISCUSSION}

Ten patients with bilateral extremity lymphedema were evaluated using the TLS staging system and ICG lymphography, found to have asymmetric severity in the individual limb, and given limb-individualized treatment involving ipsilateral VLNT and contralateral LVA simultaneously. To the best of the authors' knowledge, this study is the first case series of patients with bilateral extremity lymphedema of different severities who underwent VLNT in the advanced-stage lymphedematous extremity and LVA in the early-stage extremity. The results of this study reveal that the aforementioned procedures individualized by disease severity achieved efficacious improvement in limb circumference and episodes of cellulitis for each limb, as well as improvements in overall LYMQoL.

Methodologically, the evaluation modalities were critical for understanding and standardizing the degree of severity in bilateral lymphedematous extremities in the absence of a "normal" baseline. Lymphoscintigraphy yields useful information about proximal and intermediate lymph nodes, superficial and deep lymphatic ducts, and dermal backflow. ${ }^{1,9,23}$ ICG lymphography, specific the Dermal Backflow ICG Classification by Yamamoto et al., is a crucial additional component in the evaluation modalities, as it reveals the patency of superficial lymphatic ducts and dermal backflow patterns. ${ }^{3,24}$ We found that using the linear pattern of ICG as the indication for LVA in the extremity lymphedema with partial obstruction on lymphoscintigraphy enabled great functional recovery of the early-stage extremity lymphedema. Real-time flow dynamics and visualization of the course of lymphatics offer data that is complementary and confirmatory to the results of ICG lymphography in addition to the generalized defects visualized by lymphoscintigraphy. By utilizing both lymphoscintigraphy and ICG lymphography to classify the lymphedema severity in individual limb, the indications of VLNT and LVA for individual lymphedematous limb could be clarified and executed effectively. ${ }^{7,25,26}$

Interestingly, five patients with lower extremity lymphedema included in this study showed different progression of lymphedema between the two lower limbs after the same pelvic interventions (Table 1). ${ }^{1,27,28}$ This observation suggests that it is possible that these patients developed partial obstruction in unilateral lower extremity lymphedema but total obstruction in the other lower extremity. $^{24}$

In case 3, in which circumferential improvement after the VSLN flap transfer was not found, venous congestion with successful salvage was encountered. This patient had multiple comorbidities including history of deep venous thrombosis and chronic diabetes, which are predisposing conditions for venous thrombosis and postoperative infection. Such conditions can compromise functional recovery after VLNT.

In the LVA group, anastomoses were performed in sideto-end fashion to avoid damaging existing functional lymphatic ducts. A mean of 1.1 LVAs per limb was performed in this study to achieve success in the early-stage lymphedematous limbs without performing VLNT. ${ }^{13,14}$ Interestingly, Tourani et al. showed that the long-term patency rates of LVA in chronic lymphedema animal models are suboptimal. ${ }^{29}$ This lack of durability is hypothesized to be due to chronic inflammation and scarring of the lymphatics. This issue is not a known problem with distal VLNT for advanced-stage lymphedematous limbs, where transferred vascularized lymph nodes actively drain the lymph into the venous system by the pump mechanism, catchment effect, and gravity effects. ${ }^{5,6}$

Although durable results are typically a standard goal for surgical interventions, it may not be appropriate to suggest that only VLNT be performed for bilateral extremity lymphedema regardless of the stage of severity. First, VLNT has been shown to be indicated for advancedstage extremity lymphedema, and it is a more invasive procedure than LVA. Compared with LVA, VLNT requires intraoperative techniques of greater complexity and is associated with a higher risk for postoperative reexploration due to the nature of the multiple anastomoses and the need to maintain lymph node viability and the flap inset. ${ }^{30}$ Additionally, the submental region is the preferred donor lymph node basin, ${ }^{11,17}$ as it includes numerous sizable lymph nodes, a reliable skin paddle, and minimal risk for iatrogenic lymphedema. ${ }^{9,11,31,32}$ Bilateral LVAs for both 


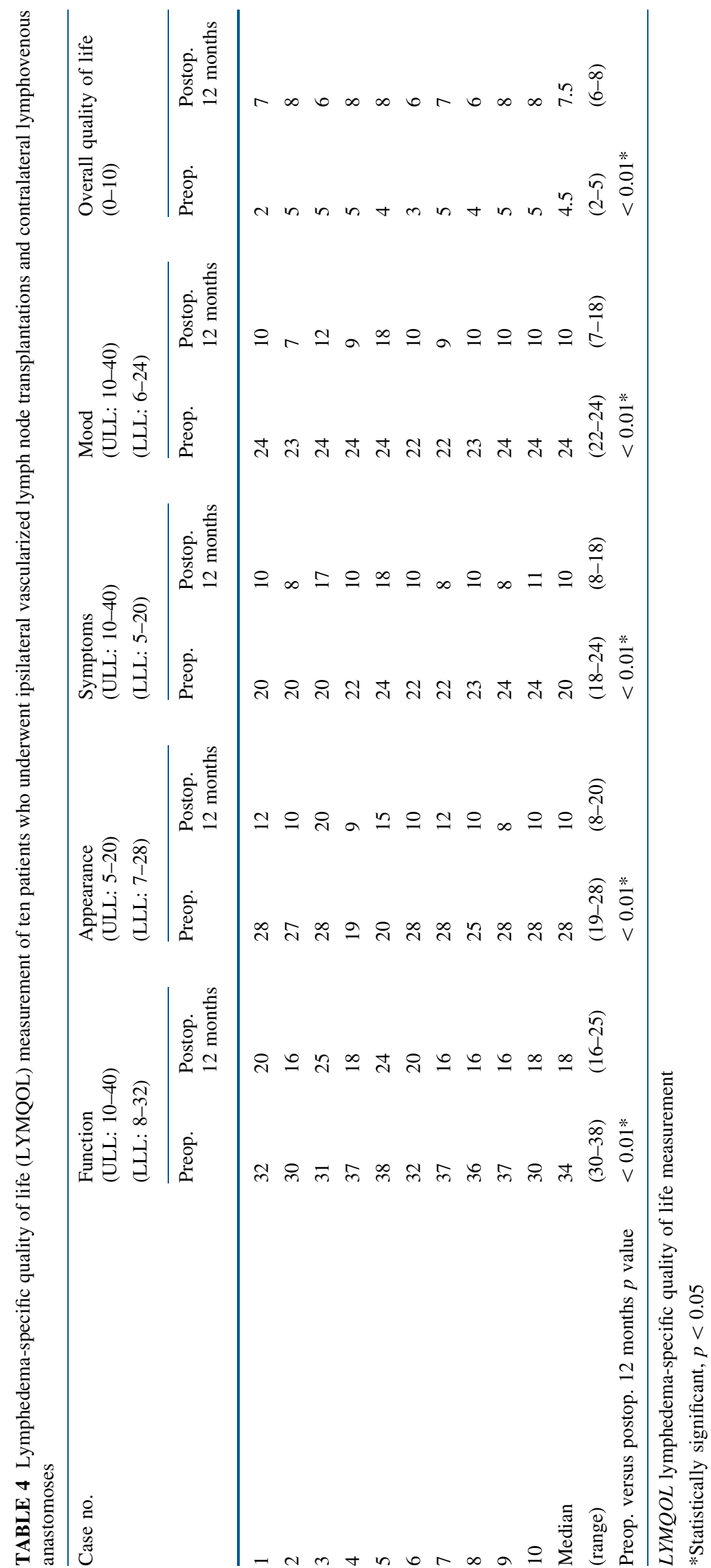


FIG. 1 Diagnosis, surgical treatment, and outcome of a 68-year-old female (case 2) who suffered from bilateral lower extremity lymphedema of different severities.

a Lymphoscintigraphy showed bilateral lymphedema, and the Taiwan Lymphoscintigraphy Staging results were more severe on the left (total obstruction) than right side (partial obstruction);

b Indocyanine green

lymphography showed patent lymphatic ducts in the right leg but diffuse dermal backflow in the left leg; c A right vascularized submental lymph node flap was harvested and transferred to left ankle with end-to-end anastomosis of the facial artery to the posterior tibial artery and end-to-end anastomosis of the facial vein to the great saphenous vein; $\mathbf{d}$ Two side-to-end lymphovenous anastomoses were performed on the right ankle; e Photographs taken preoperatively and at 19-month follow-up showed improvement in the right leg (at 4 and $3 \mathrm{~cm}$ above and below knee) and left leg (at 3 and $1.5 \mathrm{~cm}$ above and below knee). Her overall LYMQoL improved from 5 to 8 at 12-month followup
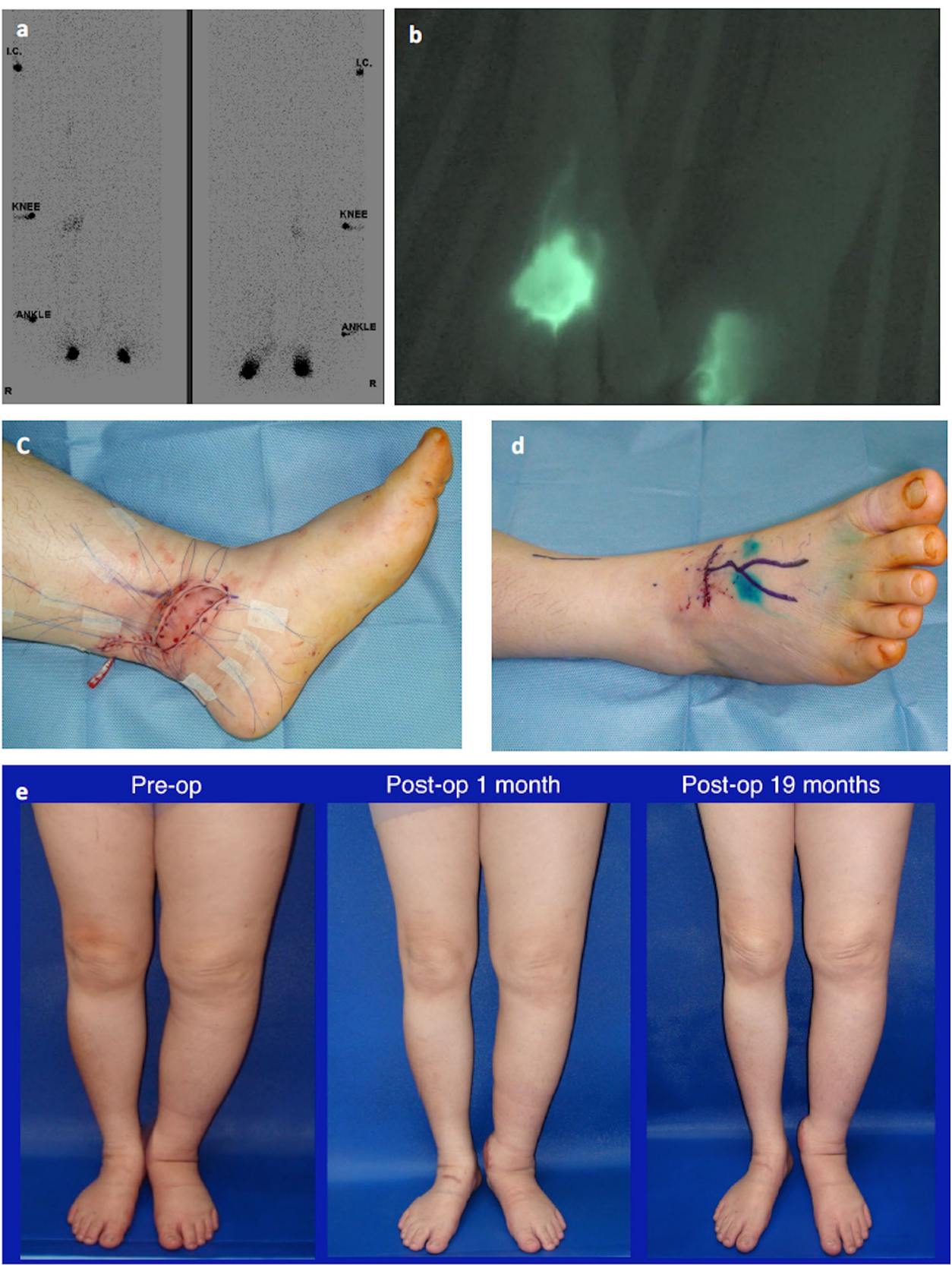

lymphedematous limbs are potentially alternative treatments for bilateral extremity lymphedema, as this does not require a second procedure (removal of the skin paddle in VLNT). This is unlikely to be an adequate solution as LVA has been shown to be less efficacious than VLNT in higher stages of lymphedema. ${ }^{11}$ Additionally, in more severe or late-stage limbs, the level of scarring, fibrosis, and inflammation in the affected limb can be severe and increase the risk that the performed LVA will damage the residual lymphatic vessels and thus of an unnecessary and ineffective procedure. Thus, it is more rational to tailor the treatment of both lymphedematous limbs on an individual basis by lymphatic severity, even in the context of bilateral extremity lymphedema with a common cause.

Proper resolution of extremity lymphedema in individual limb is of the utmost importance. Extremity lymphedema has been shown to compromise the quality of life of patients and hinder their activities. As the survival rate of cancer patients continues to improve, it will become increasingly important to strive to improve the quality of life of cancer survivors; thus, it is imperative to improve lymphedema treatment. ${ }^{10}$ All patients in this study experienced significant overall quality of life improvement. 
The major limitation of this study is its small sample size, partly reflective of limiting selection criteria. Longer follow-up times and a large number of patients are mandatory in further studies.

\section{CONCLUSIONS}

Lymphoscintigraphy and ICG lymphography can accurately differentiate the different severity in the individual limb of bilateral extremity lymphedema patients. Simultaneous ipsilateral VLNT and contralateral LVA were effective for bilateral extremity lymphedema with asymmetrical severity, resulting in improvements in circumferential measurements, episodes of cellulitis, and LYMQoL.

DATA AVAILABILITY STATEMENT The data that support the findings of this study are available from the corresponding author upon reasonable request.

DISCLOSURE Dr. Cheng received textbook royalties from Elsevier, Inc. The other authors have nothing to disclose.

OPEN ACCESS This article is licensed under a Creative Commons Attribution 4.0 International License, which permits use, sharing, adaptation, distribution and reproduction in any medium or format, as long as you give appropriate credit to the original author(s) and the source, provide a link to the Creative Commons licence, and indicate if changes were made. The images or other third party material in this article are included in the article's Creative Commons licence, unless indicated otherwise in a credit line to the material. If material is not included in the article's Creative Commons licence and your intended use is not permitted by statutory regulation or exceeds the permitted use, you will need to obtain permission directly from the copyright holder. To view a copy of this licence, visit http://creativecommons. org/licenses/by/4.0/.

\section{REFERENCES}

1. Beesley V, Janda M, Eakin E, Obermair A, Battistutta D. Lymphedema after gynecological cancer treatment: prevalence, correlates, and supportive care needs. Cancer. 2007;109(12):2607-14.

2. Ligabue MB, Campanini I, Veroni P, Cepelli A, Lusuardi M, Merlo A. Efficacy of self-administered complex decongestive therapy on breast cancer-related lymphedema: a single-blind randomized controlled trial. Breast Cancer Res Treat. 2019;175(1):191-201.

3. Chang DW. Lymphaticovenular bypass for lymphedema management in breast cancer patients: a prospective study. Plast Reconstr Surg. 2010;126(3):752-8.

4. Cheng MH, Chen SC, Henry SL, Tan BK, Lin MC, Huang JJ. Vascularized groin lymph node flap transfer for postmastectomy upper limb lymphedema: flap anatomy, recipient sites, and outcomes. Plast Reconstr Surg. 2013;131(6):1286-98.

5. Ito R, Zelken J, Yang CY, Lin CY, Cheng MH. Proposed pathway and mechanism of vascularized lymph node flaps. Gynecol Oncol. 2016;141(1):182-8.
6. Cheng MH, Huang JJ, Wu CW, et al. The mechanism of vascularized lymph node transfer for lymphedema: natural lymphaticovenous drainage. Plast Reconstr Surg. 2014;133(2):192e-8e.

7. Allen RJ, Jr., Cheng MH. Lymphedema surgery: patient selection and an overview of surgical techniques. J Surg Oncol. 2016;113(8):923-31.

8. Qiu SS, Chen HY, Cheng MH. Vascularized lymph node flap transfer and lymphovenous anastomosis for klippel-trenaunay syndrome with congenital lymphedema. Plast Reconstr Surg Glob Open. 2014;2(6):e167.

9. Cheng MH, Pappalardo M, Lin C, Kuo CF, Lin CY, Chung KC. Validity of the novel Taiwan lymphoscintigraphy staging and correlation of cheng lymphedema grading for unilateral extremity lymphedema. Ann Surg. 2018;268(3):513-25.

10. Patel KM, Lin CY, Cheng MH. A prospective evaluation of lymphedema-specific quality-of-life outcomes following vascularized lymph node transfer. Ann Surg Oncol. 2015;22(7):2424-30.

11. Engel H, Lin CY, Huang JJ, Cheng MH. Outcomes of lymphedema microsurgery for breast cancer-related lymphedema with or without microvascular breast reconstruction. Ann Surg. 2018;268(6):1076-83.

12. Pappalardo M, Patel K, Cheng MH. Vascularized lymph node transfer for treatment of extremity lymphedema: an overview of current controversies regarding donor sites, recipient sites and outcomes. J Surg Oncol. 2018;117(7):1420-31.

13. Ito $\mathrm{R}, \mathrm{Wu} \mathrm{CT}$, Lin MC, Cheng MH. Successful treatment of early-stage lower extremity lymphedema with side-to-end lymphovenous anastomosis with indocyanine green lymphography assisted. Microsurgery. 2016;36(4):310-5.

14. AlJindan FK, Lin CY, Cheng MH. Comparison of outcomes between side-to-end and end-to-end lymphovenous anastomoses for early-grade extremity lymphedema. Plast Reconstr Surg. 2019;144(2):486-96.

15. Pappalardo $\mathrm{M}$, Cheng $\mathrm{MH}$. Lymphoscintigraphy for the diagnosis of extremity lymphedema: Current controversies regarding protocol, interpretation, and clinical application. J Surg Oncol. 2020;121(1):37-47.

16. Keeley V, Sue C, Locke J, Veigas D, Riches K, Hilliam R. A quality of life measure for limb lymphoedema (LYMQOL). $J$ Lymphoedema. 2010; 5(1):26-37.

17. Cheng MH, Huang JJ, Nguyen DH, et al. A novel approach to the treatment of lower extremity lymphedema by transferring a vascularized submental lymph node flap to the ankle. Gynecol Oncol. 2012;126(1):93-8.

18. Poccia I, Lin CY, Cheng MH. Platysma-sparing vascularized submental lymph node flap transfer for extremity lymphedema. $J$ Surg Oncol. 2017;115(1):48-53.

19. Cheng MH, Lin CY, Patel KM. A prospective clinical assessment of anatomic variability of the submental vascularized lymph node flap. J Surg Oncol. 2017;115(1):43-7.

20. Visconti G, Salgarello M, Hayashi A. The recipient venule in supermicrosurgical lymphaticovenular anastomosis: flow dynamic classification and correlation with surgical outcomes. $J$ Reconstr Microsurg. 2018;34(8):581-9.

21. 30. Visconti G, Yamamoto T, Hayashi N, Hayashi A. Ultrasoundassisted lymphaticovenular anastomosis for the treatment of peripheral lymphedema. Plast Reconstr Surg. 2017;139(6):1380e-1e.

22. Yamamoto T, Koshima I. A prospective analysis of 100 consecutive lymphovenous bypass cases for treatment of extremity lymphedema. Plast Reconstr Surg. 2014;133(6):887e-8e.

23. Akita S, Mitsukawa N, Kazama T, et al. Comparison of lymphoscintigraphy and indocyanine green lymphography for the 
diagnosis of extremity lymphoedema. J Plast Reconstr Aesthet Surg. 2013;66(6):792-8.

24. Yamamoto T, Matsuda N, Doi K, et al. The earliest finding of indocyanine green lymphography in asymptomatic limbs of lower extremity lymphedema patients secondary to cancer treatment: the modified dermal backflow stage and concept of subclinical lymphedema. Plast Reconstr Surg. 2011;128(4):314e-21e.

25. Akita S, Mitsukawa N, Kuriyama M, et al. Suitable therapy options for sub-clinical and early-stage lymphoedema patients. $J$ Plast Reconstr Aesthet Surg. 2014;67(4):520-5.

26. Masia J, Pons G, Rodriguez-Bauza E. Barcelona lymphedema algorithm for surgical treatment in breast cancer-related lymphedema. J Reconstr Microsurg. 2016;32(5):329-35.

27. Hopp EE, Osborne JL, Schneider DK, Bojar CJ, Uyar DS. A prospective pilot study on the incidence of post-operative lymphedema in women with endometrial cancer. Gynecol Oncol Rep. 2016;15:25-8.

28. Loukas M, Wartmann CT, Louis RG, Jr., et al. Cisterna chyli: a detailed anatomic investigation. Clin Anat. 2007;20(6):683-8.

29. Tourani SS, Taylor GI, Ashton MW. Long-term patency of lymphovenous anastomoses: a systematic review. Plast Reconstr Surg. 2016;138(2):492-8.

30. Koide S, Lin CY, Chen C, Cheng MH. Long-term outcome of lower extremity lymphedema treated with vascularized lymph node flap transfer with or without venous complications. $J$ Surg Oncol. 2020;121(1):129-37.

31. Ciudad P, Agko M, Perez Coca JJ, et al. Comparison of long-term clinical outcomes among different vascularized lymph node transfers: 6-year experience of a single center's approach to the treatment of lymphedema. J Surg Oncol. 2017;116(6):671-682.

32. Massey MF, Gupta DK. The incidence of donor-site morbidity after transverse cervical artery vascularized lymph node transfers: the need for a lymphatic surgery national registry. Plast Reconstr Surg. 2015;135(5):939e-40e.

33. Scaglioni MF, Fontein DBY, Arvanitakis M, Giovanoli P. Systematic review of lymphovenous anastomosis (LVA) for the treatment of lymphedema. Microsurgery. 2017;37(8):947-53.
34. Silva AK, Chang DW. Vascularized lymph node transfer and lymphovenous bypass: Novel treatment strategies for symptomatic lymphedema. J Surg Oncol. 2016;113(8):932-9.

35. Koshima I, Inagawa K, Urushibara K, Moriguchi T. Supermicrosurgical lymphaticovenular anastomosis for the treatment of lymphedema in the upper extremities. J Reconstr Microsurg. 2000;16(6):437-42.

36. Koshima I, Nanba Y, Tsutsui T, Takahashi Y, Itoh S. Long-term follow-up after lymphaticovenular anastomosis for lymphedema in the leg. J Reconstr Microsurg. 2003;19(4):209-15.

37. Althubaiti GA, Crosby MA, Chang DW. Vascularized supraclavicular lymph node transfer for lower extremity lymphedema treatment. Plast Reconstr Surg. 2013;131(1):133e-5e.

38. Maldonado AA, Chen R, Chang DW. The use of supraclavicular free flap with vascularized lymph node transfer for treatment of lymphedema: A prospective study of 100 consecutive cases. $J$ Surg Oncol. 2017;115(1):68-71.

39. Akita S, Mitsukawa N, Kuriyama M, et al. Comparison of vascularized supraclavicular lymph node transfer and lymphaticovenular anastomosis for advanced stage lower extremity lymphedema. Ann Plast Surg. 2015;74(5):573-9.

40. Masia J, Pons G, Nardulli ML. Combined surgical treatment in breast cancer-related lymphedema. J Reconstr Microsurg. 2016;32(1):16-27.

41. Yamamoto $\mathrm{T}$, Yamamoto $\mathrm{N}$, Doi $\mathrm{K}$, et al. Indocyanine greenenhanced lymphography for upper extremity lymphedema: a novel severity staging system using dermal backflow patterns. Plast Reconstr Surg. 2011;128(4):941-7.

42. Yamamoto $T$, Yoshimatsu $H$, Narushima M, Yamamoto $N$, Hayashi A, Koshima I. Indocyanine green lymphography findings in primary leg lymphedema. Eur J Vasc Endovasc Surg. 2015;49(1):95-102.

Publisher's Note Springer Nature remains neutral with regard to jurisdictional claims in published maps and institutional affiliations. 